Review Article

\title{
Short Review on Types of Heat Shock Proteins and Their Fundamental Characters for Body Maintenance Together With Role in Cancer
}

${ }^{a}$ Muhammad Muneeb, ${ }^{b}$ Moazam Ali $*$, ${ }^{a}$ Tahir Sarfaraz, ${ }^{c}$ Wajid Ali, ${ }^{d}$ Zeeshan Ahmad Bhutta

\author{
${ }^{\text {a }}$ Department of Pathology, University of Agriculture Faisalabad, Pakistan \\ ${ }^{b}$ Department of Clinical Medicine and Surgery, University of Agriculture Faisalabad, Pakistan \\ 'Department of Animal Production and Technologies, Nigde Omer Halisdemir University, Turkey \\ ${ }^{\mathrm{d}}$ The Royal (Dick) School of Veterinary Studies, University of Edinburgh, Easter Bush Campus, Midlothian, EH25 9RG, \\ Scotland, United Kingdom
}

*Corresponding email: moazamali17@gmail.com

\begin{abstract}
Body of living thing is a complex machine that works on multifunctional processes and needs maintenance. Heat shock protein is a specific type of protein that cares about many normal functions of the body. These proteins have many dynamic occupations to shield the body from various diseases and also a key role in the coiling and uncoiling of proteins, prevent from apoptosis and transportation of proteins. Along with these all properties, the foremost function of these proteins is prevention from cancer and a significant role in cancer diagnosis. Commonly heat shock protein known as chaperones and a wide range of their types have been discovered with their functions as well. Recently many scientists are working on additional investigation of heat shock proteins. This review concludes some basic types of heat shock proteins and their elegant purposes and also providing an open eye for new scientist about a further investigation of heat shock protein.
\end{abstract}

\begin{tabular}{l}
\multicolumn{1}{c}{ Article Info } \\
\hline Article history: \\
Received date: 18 July 2020 \\
Accepted date: 21 August 2020 \\
\hline Keywords: \\
HSP \\
Cancer \\
Apoptosis \\
HSP types \\
\hline
\end{tabular}

\section{Introduction}

The escalating human race has resulted in the emergence of new diseases via the imbalance between the food supply chain, global warming, and poor health status in underdeveloped countries. One of the most abruptly increasing condition in the human population with its devastating effects is "Cancer". In 2018, about 0.6 million individuals died in the United States just because of cancer, by taking into account the alarming situation, the scientific community and research institutes are actively working to trump up an adequate solution to this disaster. The role of heat shock proteins has been proved to be effective for controlling and minimizing the effect of this catastrophe. Heat shock proteins coherently termed stress proteins, naturally present in all the cells of an organism (Lindquist, 1986). These heat shock proteins are contemporaneous in complex state among all species. A special type of heat shock proteins is "Chaperones" their chief role is the coiling and uncoiling of proteins besides they also implement other functions like protection of cells from apoptosis, cell signaling, the formation of multicomplex proteins and transportation and storing of protein in subcellular compartments. Heat-shock proteins (HSPs), or stress proteins, are present in all organisms and all cells. Recent studies have shown that heat shock proteins have exhibit antigen presentation by transferring antigen peptides to major histocompatibility complex class 1 and class 2 molecules ( $\mathrm{Li}$ et al., 2002). Moreover, heat shock proteins help to strengthen the immune system like macrophages and the dendritic cells for presentation of antigen (Srivastava, 2002). Heat shock proteins have the variable molecular size of 10 to $100 \mathrm{kDa}$ (kilo Dalton) or more and they reside in different cellular compartments. $\mathrm{Xu}$ (2002) highlighted the accumulation of heat shock proteins in various locations within the cell such as HSP10, 60 and 75 in mitochondria while the other heat shock proteins are present on cytoplasm, nucleus, endoplasmic reticulum and cytosol under different physiological conditions. This review overlooks the different types of HSP's and 
their physiological activity within the body and how they fruitfully take part in diagnosing the cancer and its treatment.

\section{Types of Heat Shock Protein (Hsp) and Their Impressive Functions}

HSP has 10 to 30 types having a molecular weight ranging from 15 to $43 \mathrm{kDa}$ (kilo Dalton) and recognized as heat shock protein $\beta$ (HSPBs) with the chaperoning effect in the embryonic development process. HSPBs maintain homeostasis with the cytoskeleton proteins to preclude damage and conserving the cellular function. HSPB-1, HSPB-6, HSPB-7, and HSPB-12 have a characteristic responsibility in respiratory morphology development. The absence of HSPBs in cardiovascular diseases leads to the development of squeal like cardiomyopathy and lethal myopathy. (Juo et al., 2016) (Ke et al., 2011) (Rosenfeld et al., 2013) Heat shock proteins with a molecular size of 15-30 kDa are known as small HSP and these are 9 in numbers present in mammals (Mounier \& Arrigo, 2002). HSP-27 and HSP-25 present in mice and recently identified HSP-22 is also categorized in small molecular size heat shock proteins. Other members of this family include $\alpha \mathrm{A}$ - and $\alpha \mathrm{B}$ crystallins, Hsp B different types like HSP $\beta-2$, HSP $\beta-3$, НSP $\beta-7$, HSP $\beta-8$, and HSP $\beta-9$. HSP10 is released from the fetal placental unit and mainly suppresses the maternal immune system (Noonan et al., 1979). HSP10 is consisted of 101 amino acids and also used as a biomarker in endometrial cancer (Dubé et al., 2007). In mitochondria, HSP10 has a primary function in the protein folding which is supplied by the adenosine triphosphate (ATP) (Meyer et al., 2003). HSP10 is produced as a by-product during the neoplastic cell proliferation process and known as the growth factor of the cell and it plays an important role in tumor growth (Quinn et al., 1990). Human small HSPs is consist of 105 to 205 amino acids a crystalline domain which are 80 homologous and sequenced residues (Ingolia \& Craig, 1982). This crystalline domain is much conserved and has an identity of $38 \%$ to $60 \%$ amino acids (Mounier \& Arrigo, 2002). This crystalline structure can be changed by different factors like $\mathrm{pH}$, Calcium ions, ionic strength and temperature. The structure of heat shock protein 16.5 has a crystalline domain and its basic function is similar to chaperone which helps in folding and miss-folding of proteins (Bertz et al., 2010). The small HSPs have a clear effect on the function of the enzymatic activity of myosin. At $43^{\circ} \mathrm{C}$ for 30 minutes, the crystalline affects ATPase performance that prevents the aggregation of protein at the same place and by maintaining the ATPase performance that involves the generation of myofibrils without myosin unfolding and muscle performance under different stress and in case of access exercise (Melkani et al., 2006). HSP-50 and HSP-40 are present in the cytosol and acts as the co-chaperone of HSP 70 which helps in protein coiling and uncoiling. HSP40 has a size of $43 \mathrm{kDa}$ and has a different type of amino acid sequence with having different $\mathrm{N}$ terminal lengths. These heat shock proteins also have acrystallin domains and extension of $\mathrm{C}$ terminal with a high amount of I-X-I/V motifs (Ghosh et al., 2005). The HSP47 is also involved in the folding and unfolding of protein along with its involvement in atherosclerosis. HSP47 has the distinctive and pinpoint expression in the collagenous regions of atherosclerosis. The cells which are involved in procollagen expression are also intricate in the expression of HSP47 stimulated by the low density of lipoprotein and heat shock protein in the smooth muscle cells. It has been researched that HSP47 is an important part of the coronary atheroma of humans. HSP47 is also required not only for the transportation of collagen protein within cells as well as for the folding into the tertiary structure. HSP47 is present in the endoplasmic reticulum and its function is to support the folding of collagen protein and its release into the extracellular environment. The HSP60 is present in mitochondria having a size of $970 \mathrm{kDa}$ which is a large heterooligomer protein complex known as TCP ring with several other proteins that have an important role in protein formation and structure. Besides HSP-60 beneficial effects, it causes the development of rheumatoid arthritis and systemic sclerosis in humans whereas arthritis and insulin-dependent diabetes mellitus in the mice. HSP70 belongs to the family of GRP78 (Glucose response protein 78) located in the cytosol, helps in the formation of protein, folding, transportation as well as a leading role in the prevention of protein degradation in the endoplasmic reticulum. The expression of HSP70 is done by physical exercise (Banfi et al., 2004). The expression of HSP70 and HSP90 is variable and depends on different individual abilities of athletic and regulation of these proteins is done in the response of heat acclimation (McClung et al., 2008). The HSP70 plays the role of a chaperone as in the formation, 
transportation of newly synthesized protein into the cell and in case of denatured proteins. It also helps the native proteins in folding and the stabilization of their functional structure (Ogawa et al., 2011). Along with HSP70, their modification with carbohydrates also helps in the GRP78 (Glucose response protein 78) to maintain the structure of native proteins and folding of these native proteins into their functional structure. GRP78 has a supreme ability to resist the ultraviolet rays and form cisplatin which is a very famous anticancerous drug. HSP90 is present in the cytosol and nucleus of cells (Shastry et al., 2002). When muscles are under load which increases the muscle damage chances and causes different problems like muscle soreness. It elevates the hot conditions and due to its elevation, HSP72 and HSP90 expression increases (Tuttle et al., 2015). HSP90 has also a key role in the folding of myosin and the construction of sarcomere (Du et al., 2008) (Etard et al., 2007) (Hawkins et al., 2008) (Srikakulam et al., 2008). The structure of HSP90 consists of the N-terminal binding of ATP, substrate interacting middle domain, and C-terminal dimerization domain (Jackson, 2012). Due to the binding of ATP to the N-terminal ATP binding domain, the regulation of myosin filament construction and the myofibrillogenesis is done by HSP90 (Hawkins et al., 2008). HSP90 can bind with different types of receptors in a specific manner such as binding with steroid receptors, actin microfilaments, intermediate filaments, microtubules, and protein kinases. Besides these, HSP90 is an active component of glucocorticoid receptors which are found in the form of several protein complexes. HSP40 and HSP70 act as co-chaperone with heat shock protein 90 . HSP90s are also present in the cytoplasm as well as in endoplasmic reticulum which play significant features in the folding of newly synthesized proteins. HSP100 is present in the cytoplasm of the cell and acts as co-chaperone with the collaboration of different heat shock proteins like HSP40, HSP70 and HSP90 and these combinations play a vital role in the aggregation and refolding of proteins. HSP110 is located in the cytosol and the nucleus of cells (Dimauro et al., 2016). The HSP110 and HSP70 have a distinctive loop structure that helps the immune system to produce an immune response in different conditions (Zuo et al., 2016). HSP110 with the collaboration of HSP70 and GRP79 helps in the folding of protein structure and survival of cells in different stress conditions (Hartl, 1996) (Gething \&
Sambrook, 1992).

\section{Heat Shock Protein Role in Cancer}

HSP90 is the type of molecular chaperone which has a function in the stability and ultimate functioning of mutated cells and overexpressed signal proteins for the better growth of cancer cell or the survival of the cancerous cell. HSP90 includes various types of proteins such as p53, Akt, Raf-1, HIF-1 $\alpha$, HER/2Neeu (ErbB2) and Bcr-Abl. The inhibitory protein of HSP90 causes the loss of stability and degradation process of different types of HSP90 such as allylamino-17 demethoxygeldanamycin (17AAG) which is inhibitory HSP90 protein. The HSP90 fraction is present on cell surfaces which has a role in the progression of melanoma. Antibodies and cell impermeable HSP90 cause the inhibition of these cell surface fractions of HSP90 and block the invasion and cell progression as well as the metastasis process in vivo. So that, HSP90 cell surface proteins have an intense role in the tumor metastasis and can cause interaction with the intracellular function. HSP27 is the type of heat shock protein that has a role as a chaperone which not ATP dependent and discovered for the refolding of damaged proteins (Kim et al., 1998). HSP27 mostly located in the cytosol and possess properties like antioxidant and against apoptotic activity (Simioni et al., 2009). On the other side, heat shock protein has a role in cell growth, cytoskeleton architecture, cell migration and as well as in the progression of the tumor. It is the reason that HSP27 has protective and counter protective properties in case of different diseases (Khalil et al., 2011). This type of heat shock protein is different from the other heat shock proteins due to its response in case of stress. HSP27 also responds to oxidative stress as well as chemical stress. In the case of oxidative stress, the HSP27 plays a role as an antioxidant by reducing the amount of reactive oxygen in response to increased glutathione level and lowered iron concentration into the cell. HSP27 show anti-apoptotic activity by the interaction with dependent and independent apoptotic pathway related to mitochondria. It is also stated that HSP27 is also involved against the apoptotic activity by inhibiting the caspase-dependent apoptotic process (Havasi et al., 2008). Apart from its presence in the cytosol HSP27 is also present in the intra and extracellular environment. Various studies had shown that in different categories of cancer like brain, ovarian, prostate and breast cancer, there was an increase in the amount of HSP27 (Calderwood, 2010). In Brain 
tumors case study, cytoplasmic immunoreactivity of HSP27 had to be found in meningiomas. HSP27 has been detected in every case study of meningioma tissues. Even overexpression has been seen in the cases of meningiomas (Assimakopoulou, 2000). HSP27 is also correlated with cancer of glial cells and with the Ki-67 index (Schmitt et al., 2007). In the case of colorectal cancer, HSP27 overexpression and accumulation also reduce the apoptotic mechanism which is induced by the alkylating agents (Graner et al., 2007). Recent research has shown that in the case of prostate cancer, the overexpression of HSP27 has been analyzed as compared with the control group (MIYAKE et al., 2006). As it was analyzed by ELISA, the HSP27 level is also found in tumor microenvironment and serum. This overexpression of HSP27 is also noted in breast cancer as compared to the control group (Banerjee et al., 2011). Interesting results had been obtained that the breast tumor cells produce more HSP27 level rather than serum (Mischak et al., 2010).

\section{Conclusions}

Heat shock proteins coherently called as chaperone, with a chief role in the body is the maintenance of the normal structure of the protein. Recent studies have shown that heat shock protein has a role in cell protection from abnormal functions like oxidation, apoptosis, and formation of complex protein structures. Heat shock proteins are classified into different groups, according to the function they perform like identification of several diseases and cancer diagnosis. This review throws light on the functioning of heatshock proteins in neonatal and later ages according to the types. Further research studies are needed to expressively declare heat shock proteins as a tool for the treatment of cancer and other diseases.

\section{Conflicts of Interest}

No conflict of interest.

\section{Funding}

This research received no external funding.

\section{References}

Assimakopoulou, M. (2000). Human meningiomas: immunohistochemical localization of progesterone receptor and heat shock protein 27 and absence of estrogen receptor and PS2. Cancer Detection and Prevention, 24(2), 163-168.
Banerjee, S., Lin, C. F. L., Skinner, K. A., Schiffhauer, L. M., Peacock, J., Hicks, D. G., ... \& Langstein, H. N. (2011). Heat shock protein 27 differentiates tolerogenic macrophages that may support human breast cancer progression. Cancer research, 71(2), 318-327.

Banfi, G., Dolci, A., Verna, R., \& Corsi, M. M. (2004). Exercise raises serum heat-shock protein 70 (Hsp70) levels. Clinical Chemistry and Laboratory Medicine (CCLM), 42(12), 1445-1446.

Bertz, M., Chen, J., Feige, M. J., Franzmann, T. M., Buchner, J., \& Rief, M. (2010). Structural and mechanical hierarchies in the $\alpha$-crystallin domain dimer of the hyperthermophilic small heat shock protein Hsp16. 5. Journal of molecular biology, 400(5), 1046-1056.

Calderwood, S. K. (2010). Heat shock proteins in breast cancer progression-a suitable case for treatment? International Journal of Hyperthermia, 26(7), 681-685.

Dimauro, I., Mercatelli, N., \& Caporossi, D. (2016). Exercise-induced ROS in heat shock proteins response. Free Radical Biology and Medicine, 98, 46-55.

Du, S. J., Li, H., Bian, Y., \& Zhong, Y. (2008). Heatshock protein $90 \alpha 1$ is required for organized myofibril assembly in skeletal muscles of zebrafish embryos. Proceedings of the National Academy of Sciences, 105(2), 554-559.

Dubé, V., Grigull, J., DeSouza, L. V., Ghanny, S., Colgan, T. J., Romaschin, A. D., \& Siu, K. M. (2007). Verification of endometrial tissue biomarkers previously discovered using mass spectrometry-based proteomics by means of immunohistochemistry in a tissue microarray format. Journal of proteome research, 6(7), 26482655 .

Etard, C., Behra, M., Fischer, N., Hutcheson, D., Geisler, R., \& Strähle, U. (2007). The UCS factor Steif/Unc-45b interacts with the heat shock protein Hsp90a during myofibrillogenesis. Developmental biology, 308(1), 133-143.

Gething, M. J., \& Sambrook, J. (1992). Protein folding in the cell. Nature, 355(6355), 33-45.

Ghosh, J. G., Estrada, M. R., \& Clark, J. I. (2005). Interactive domains for chaperone activity in the 
small heat shock protein, human $\alpha \mathrm{B}$ crystallin. Biochemistry, 44(45), 14854-14869.

Graner, M. W., Cumming, R. I., \& Bigner, D. D. (2007). The heat shock response and chaperones/heat shock proteins in brain tumors: surface expression, release, and possible immune consequences. Journal of Neuroscience, 27(42), 11214-11227.

Hartl, F. U. (1996). Molecular chaperones in cellular protein folding. Nature, 381(6583), 571-580.

Havasi, A., Li, Z., Wang, Z., Martin, J. L., Botla, V., Ruchalski, K., ... \& Borkan, S. C. (2008). Hsp27 inhibits Bax activation and apoptosis via a phosphatidylinositol 3-kinase-dependent mechanism. Journal of Biological Chemistry, 283(18), 12305-12313.

Hawkins, T. A., Haramis, A. P., Etard, C., Prodromou, C., Vaughan, C. K., Ashworth, R., ... \& Pearl, L. H. (2008). The ATPase-dependent chaperoning activity of Hsp90a regulates thick filament formation and integration during skeletal muscle myofibrillogenesis. Development, 135(6), 11471156.

Ingolia, T. D., \& Craig, E. A. (1982). Drosophila gene related to the major heat shock-induced gene is transcribed at normal temperatures and not induced by heat shock. Proceedings of the National Academy of Sciences, 79(2), 525-529.

Jackson, S. E. (2012). Hsp90: structure and function. In Molecular chaperones (pp. 155-240). Springer, Berlin, Heidelberg.

Juo, L. Y., Liao, W. C., Shih, Y. L., Yang, B. Y., Liu, A. B., \& Yan, Y. T. (2016). HSPB7 interacts with dimerized FLNC and its absence results in progressive myopathy in skeletal muscles. Journal of cell science, 129(8), 1661-1670.

Ke, L., Meijering, R. A., Hoogstra-Berends, F., Mackovicova, K., Vos, M. J., Van Gelder, I. C., ... \& Brundel, B. J. (2011). HSPB1, HSPB6, HSPB7 and HSPB8 protect against RhoA GTPase-induced remodeling in tachypaced atrial myocytes. PloS one, 6(6), e20395.

Khalil, A. A., Kabapy, N. F., Deraz, S. F., \& Smith, C. (2011). Heat shock proteins in oncology: diagnostic biomarkers or therapeutic targets?. Biochimica et
Biophysica Acta (BBA)-Reviews on Cancer, 1816(2), 89-104.

Kim, K. K., Kim, R., \& Kim, S. H. (1998). Crystal structure of a small heat-shock protein. Nature, 394(6693), 595-599.

Li, Z., Menoret, A., \& Srivastava, P. (2002). Roles of heat-shock proteins in antigen presentation and cross-presentation. Current opinion in immunology, 14(1), 45-51.

Lindquist, S. (1986). The heat-shock response. Annual Review of Biochemistry, 55, 1151-1191.

McClung, J. P., Hasday, J. D., He, J. R., Montain, S. J., Cheuvront, S. N., Sawka, M. N., \& Singh, I. S. (2008). Exercise-heat acclimation in humans alters baseline levels and ex vivo heat inducibility of HSP72 and HSP90 in peripheral blood mononuclear cells. American Journal of Physiology-Regulatory, Integrative and Comparative Physiology, 294(1), R185-R191.

Melkani, G. C., Cammarato, A., \& Bernstein, S. I. (2006). $\alpha \mathrm{B}$-crystallin maintains skeletal muscle myosin enzymatic activity and prevents its aggregation under heat-shock stress. Journal of molecular biology, 358(3), 635-645.

Meyer, A. S., Gillespie, J. R., Walther, D., Millet, I. S., Doniach, S., \& Frydman, J. (2003). Closing the folding chamber of the eukaryotic chaperonin requires the transition state of ATP hydrolysis. Cell, 113(3), 369-381.

Mischak, H., Allmaier, G., Apweiler, R., Attwood, T., Baumann, M., Benigni, A., ... \& Coon, J. J. (2010). Recommendations for biomarker identification and qualification in clinical proteomics. Science translational medicine, 2(46), 46ps42-46ps42.

Miyake, H., Muramaki, M., Kurahashi, T., Yamanaka, K., Hara, I., \& Fujisawa, M. (2006). Enhanced expression of heat shock protein 27 following neoadjuvant hormonal therapy is associated with poor clinical outcome in patients undergoing radical prostatectomy for prostate cancer. Anticancer research, 26(2B), 1583-1587.

Mounier, N., \& Arrigo, A. P. (2002). Actin cytoskeleton and small heat shock proteins: how do they interact?. Cell stress \& chaperones, 7(2), 167. 
Noonan, F. P., Halliday, W. J., Morton, H., \& Clunie, G. J. A. (1979). Early pregnancy factor is immunosuppressive. Nature, 278(5705), 649-651.

Ogawa, K., Seta, R., Shimizu, T., Shinkai, S., Calderwood, S. K., Nakazato, K., \& Takahashi, K. (2011). Plasma adenosine triphosphate and heat shock protein 72 concentrations after aerobic and eccentric exercise. Exerc Immunol Rev, 17, 136149.

Quinn, K. A., Athanasas-Platsis, S., Wong, T. Y., Rolfe, B. E., Cavanagh, A. C., \& Morton, H. (1990). Monoclonal antibodies to early pregnancy factor perturb tumour cell growth. Clinical \& Experimental Immunology, 80(1), 100-108.

Rosenfeld, G. E., Mercer, E. J., Mason, C. E., \& Evans, T. (2013). Small heat shock proteins Hspb7 and Hspb12 regulate early steps of cardiac morphogenesis. Developmental biology, 381(2), 389-400.

Schmitt, E., Gehrmann, M., Brunet, M., Multhoff, G., \& Garrido, C. (2007). Intracellular and extracellular functions of heat shock proteins: repercussions in cancer therapy. Journal of leukocyte biology, 81(1), 15-27.

Shastry, S., Toft, D. O., \& Joyner, M. J. (2002). HSP70 and HSP90 expression in leucocytes after exercise in moderately trained humans. Acta physiologica scandinavica, 175(2), 139-146.
Simioni, M. B., De Thonel, A., Hammann, A., Joly, A. L., Bossis, G., Fourmaux, E., Bouchot,A., Landry, J., Piechaczyk, M., \& Garrido, C. (2009). Heat shock protein 27 is involved in SUMO-2/3 modification of heat shock factor 1 and thereby modulates the transcription factor activity. Oncogene, 28(37), 3332-3344.

Srikakulam, R., Liu, L., \& Winkelmann, D. A. (2008). Unc 45 b forms a cytosolic complex with Hsp90 and targets the unfolded myosin motor domain. PloS one, 3(5), e2137.

Srivastava, P. (2002). Roles of heat-shock proteins in innate and adaptive immunity. Nature reviews immunology, 2, 185-194.

Tuttle, J. A., Castle, P. C., Metcalfe, A. J., Midgley, A. W., Taylor, L., \& Lewis, M. P. (2015). Downhill running and exercise in hot environments increase leukocyte Hsp72 (HSPA1A) and Hsp90 $\alpha$ (HSPC1) gene transcripts. Journal of Applied Physiology, 118, 996-1005.

$\mathrm{Xu}$, Q. (2002). Role of heat shock proteins in atherosclerosis. Arteriosclerosis, Thrombosis, and Vascular Biology, 22, 1547-1559.

Zuo, D., Subjeck, J., \& Wang, X. Y. (2016). Unfolding the role of large heat shock proteins: new insights and therapeutic implications. Frontiers in immunology, 7, 75 .

How to cite: Muneeb, M., Ali, M., Sarfaraz, T., Ali, W., \& Bhutta, Z. A. (2020). Short Review on Types of Heat Shock Proteins and Their Fundamental Characters for Body Maintenance Together With Role in Cancer. Himalayan Journal of Science and Technology, 3-4, 103-108. 